\section{DGI-029 EFFICACY AND SAFETY OF PROPRANOLOL IN INFANTILE HAEMANGIOMA}

doi:10.1136/ejhpharm-2013-000276.295

L García López, S Fernández Cañabate, V Cabezas Martín, V Martínez Santana, M Izquierdo Navarro, C Matallana Martín, A Salvador Palacios, AM López González, T Sànchez Sánchez. Hospital Clínico Universitario, Pharmacy, Valladolid, Spain

Background Infantile haemangiomas are common vascular tumours in children. Only 10-15\% should be treated due to any vital, functional or aesthetic complication. Oral corticosteroids have been the primary treatment of choice. However, excellent outcomes have been reported with propranolol, and using it as first-line treatment is still a matter of debate.

Purpose To evaluate the short-term efficacy and safety of propranolol in the treatment of infantile haemangioma.

Materials and Methods A retrospective study was carried out in the Pharmacy Service of the Hospital Clínico Universitario de Valladolid between June 2009 and August 2012. All patients with infantile haemangioma treated with propranolol during the study period were included.

Results 32 patients (20 female) were treated with propranolol for an average of 9 months. Patients started treatment at an average age of 6 months (1-15). 9/32 of the haemangiomas had segmental distribution and $23 / 32$ were located in the head and neck. 4/32 patients were previously been treated with oral corticosteroids with little improvement. 8/32 of patients achieved complete remission after $11 \pm 5$ months of treatment. One of these patients had to discontinue treatment due to an increase in the size of the lesion. In the remaining patients the use of propranolol accelerated the involution of the haemangiomas and decreased colour, brightness and growth. Adverse events were mild and self-limiting. Only 2 patient discontinued treatment due to hypotension.

Conclusions Only a quarter of patients achieved complete remission.

The average duration of treatment until complete remission was 11 months.

Only one patient didn't achieve any improvement.

The use of propranolol is a safe alternative for treating haemangiomas.

No conflict of interest.

\section{DGI-030 EFFICACY AND SAFETY OF TELAPREVIR IN PATIENTS WITH CHRONIC HEPATITIS C VIRUS GENOTYPE 1}

doi:10.1136/ejhpharm-2013-000276.296

'D Guerra Estevez, 'MP Quesada Sanz, 'JJ Ramos Báez, 'P Villanueva Jiménez, ${ }^{2} \mathrm{~F}$ Rodriguez Muñoz. ${ }^{1}$ Hospital Punta Europa, Pharmacy, Algeciras, Spain; ${ }^{2}$ Hospital Punta Europa, Digestive, Algeciras, Spain

Background The addition of telaprevir to standard treatment considerably improves response rates and allows the duration of treatment to be reduced in a significant number of patients.

Purpose To assess the efficacy and safety of telaprevir in combination with peginterferon alfa-2b and ribavirin (RBV) in patients with hepatitis $\mathrm{C}$ virus genotype 1 (HCV).

Materials and Methods Retrospective observational study of patients mono-infected with HCV genotype 1, treatment-naive and pretreated, who started treatment with telaprevir. The follow-up period was 24 weeks. Relapsed patients were defined as those with undetectable viral load at the end of treatment but detectable at 24 weeks' follow-up, partial responders as $\geq 2 \log 10$ decline in viral RNA at week 12 but without undetectable viral load at week 24 and null responders as $<2 \log 10$ decline in viral RNA at week 12 . Some of the variables were: degree of fibrosis, basal viral load, at week 4 and at week 12 (IU/ml), duration of treatment (weeks), basal dose of RBV (mg/day), basal haemoglobin at week 4 and at week $12(\mathrm{mg} / \mathrm{dl})$, need for blood transfusions and support with erythropoietin and skin toxicity (mild/moderate/severe).

Results We included 16 patients ( $81.3 \%$ men and $18.8 \%$ women). 15 patients presented undetectable viral load at weeks 4 and 12, reducing the duration of treatment to 24 weeks. RBV dose was reduced in 6 patients and 2 patients started with a dose of $600 \mathrm{mg}$, in both cases without compromising treatment success. 7 patients had anaemia, of whom 2 required transfusions and erythropoietin. 12 cases had skin toxicity ( 8 mild, 3 moderate and 1 severe with subsequent interruption of treatment at week 4).

Conclusions The data confirm those reported in the ILLUMINATE study, with high rates of rapid virological response and reduction of treatment from 48 to 24 weeks, but with a higher rate of skin toxicity although mostly mild to moderate.

No conflict of interest.

\section{DGI-031 EFFICACY OF ORAL THALIDOMIDE IN PATIENTS WITH RECURRENT GASTROINTESTINAL BLEEDING}

doi:10.1136/ejhpharm-2013-000276.297

C García Fernandez, S Ruiz Fuentes, C Gómez Peña, S Belda Rustarazo, C Fernandez López, C Medarde Caballero, D Blanquez Martinez, A Caballero Romero. Hospital San Cecilio, Farmacia Hospitalaria, Granada, Spain

Background Recurrent gastrointestinal bleeding caused by angiodysplasia, and not responding to standard treatment, currently lacks effective medical treatment.

Purpose To evaluate the efficacy of oral thalidomide in patients with gastrointestinal bleeding from angiodysplasia refractory to other treatments.

Materials and Methods Retrospective study for a year including all patients with recurrent gastrointestinal bleeding treated with oral thalidomide manufactured in the pharmacotechnic unit of a tertiary level hospital.

The information was obtained from the outpatient dispensing programme Farmatools, the Paracelso pharmacotechnics programme, and by reviewing medical records from the hospital 1, Archinet.

For each patient we extracted the diagnosis, treatments used for gastrointestinal bleeding, line and duration of treatment with thalidomide and transfusion requirements after treatment.

Results In the study period were identified 3 patients for whom the Digestive Service ordered thalidomide capsules $100 \mathrm{mg}$. The patients had not responded to standard treatments such as argon gas sessions and octreotide. They were introduced to thalidomide $100 \mathrm{mg}$ daily for 4 months. One of them discontinued treatment for intolerance and the other 2 completed the course. There was a decrease in the number of transfusions after treatment with thalidomide in all 3 cases.

Conclusions Thalidomide appears to be a therapeutic alternative to consider when treating gastrointestinal bleeding caused by angiodysplasia in cases where there is no response to conventional treatments. One impediment to this treatment option is intolerance in some patients, leading to treatment discontinuation. Thalidomide is less aggressive than other drugs used and appears to decrease patients' transfusion requirements.

No conflict of interest.

\section{DGI-032 EPIDEMIOLOGICAL MONITORING OF PEMETREXED USE IN MALIGNANT PLEURAL MESOTHELIOMA: A TOOL OF LOCAL DECISION MAKING}

doi:10.1136/ejhpharm-2013-000276.298

'L Esposito, ${ }^{1} \mathrm{AC}$ Palozzo, ${ }^{2} \mathrm{~A}$ Jirillo, ${ }^{3} \mathrm{G}$ Pasello, ${ }^{3} \mathrm{~A}$ Favaretto, ${ }^{1} \mathrm{~F}$ Paganelli. ${ }^{1 / s t i t u t o}$ Oncologico Veneto IRCCS, Farmacia, Padova, Italy; ${ }^{2}$ Istituto Oncologico Veneto IRCSS, Anticancer Drugs Evaluation Center, Padova, Italy; ${ }^{3}$ stituto Oncologico Veneto IRCSS, Oncologia Medica 2, Padova, Italy 